\title{
Probability learning to escape from shock'
}

MICHAEL GOLE, GREgORY L. BELENKY, RIGHARD G. BOUGHER, ROBERT N. FERMAMDEZ AND DAVID L. MYERS

YALE UNIVERSITY

\begin{abstract}
Abatract
Probability learning to escape from shock was investigated in 36 female albino rats. The independent variable was the intensity of shock. All Ss were run in a T-maze using a correction procedure. One side of the $T,\left(A_{1}\right)$, was shock-free with probability, $\pi=.667$ while the other $\left(A_{2}\right)$ side was shock-free with probability $(1-\pi)=.333$. The major findings were: (a) $p\left(A_{1}\right)$ exceeded $\pi$ increasingly with increasingly severe shock, (b) positive recency was present throughout training and (c) response speed increased with increasing shock levels and was greater to the $A_{1}$ than the $A_{2}$ side.

\section{Problem}

Although the joint effects of magnitude and probability of reward on choice behavior have received considerable attention using human Ss (Myers \& Atkinson, 1964), evidence concerning the joint effects of these variables is rare in the animal literature.

Uhl (1963) found that with sufficient training, rats exceed $\pi$ even using a correction procedure, which earlier studies had indicated produced probability matching (Estes, 1959). Uhl's results indicate further that incentive differences are found only early in training.

In a somewhat unorthodox situation, Karsh \& Suppes (1964) found that rats would match $\pi$ to escape from shock when a modified correction procecture is used.

The present study was designed to provide data on the effects of varying shock intensity on choice behavior within a probability learning context. By analogy with human experiments, (Myers \& Atkinson, 1964), it was hypothesized that increasing shock levels would increase the probability of choosing the more frequently reinforced (shock-free) side with probability of reinforcement held constant.

\section{Method}

The Ss were 36 naive female albino rats, which were received from the supplier and run in three replication groups of 12. Each group of 12 rats was divided into three equal groups (High, Medium, and Low shock). A total of 150 trials was run for each S, 10 trials each day on consecutive days. All rats were maintained on ad libitum schedules of food and water throughout the experiment.

The apparatus consisted of a T-maze with a series of metal bars raised off the floor of the maze and wired to a Lehigh Valley grid scrambler. The T-maze measured (inside dimensions) $4-1 / 4$ in high and 4-3/4 in wide. The stem of the $T$ was 16 in long and

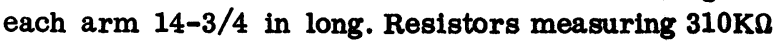
were placed in series with a $1780 \mathrm{~V}$ power supply and the grid scrambler in order to reduce current delivered to the rat. The T-maze was equipped with photocells at the beginning of the safe portion of each arm of the $T$, located $3 / 34$ in into each arm (as measured from the stem). A microswitch triggered a relay with the opening of the start box door, starting both a clock and the shock at the same time.

A random reinforcement schedule generated for each $\mathrm{S}$ on an IBM 7094 computer, provided the schedule for deciding which arm of the $T$ would be "safe" on each trial. For half of the Ss in each group the right side was safe with probability, $\pi=.667$ and the left side safe with probability, $(1-\pi)=.333$. For the other half of the Ss these assignments were reversed. Shock levels were established using behavioral criteria on nonexperimental animals. The low shock level was set at the lowest setting at which the rats would show agitated running in the maze. The high shock was set somewhat arbitrarily at a level evoking extreme agitation, squealing and rapid running. The medium level represented the "mean"' of the high and low values.

\section{Besults and Disenesion}

As indicated by Fig. 1, learning is rapid with the mean values exceeding $\pi$ in all groups after 40 trials. Measured over the final 50 trials, this overshooting was significant beyond the .001 level for the high and medium shock groups $(t=4.01$ and $t=16.67$ with $33 \mathrm{df}$ for the two groups, respectively), but the overshooting was not significant for the low shock group $(t=1.74$ with $33 \mathrm{df}$; $.05<p<.10)$. Over the 50 final trials the three groups differed significantly from each other ( $F=3.41$ with 2 and $33 \mathrm{df} ; .01<\mathrm{p}<.05$ ), indicating that increasing shock intensity was affecting choice performance.

In order to obtain an indication of the tendency of Ss in the various groups to become "absorbed" on the

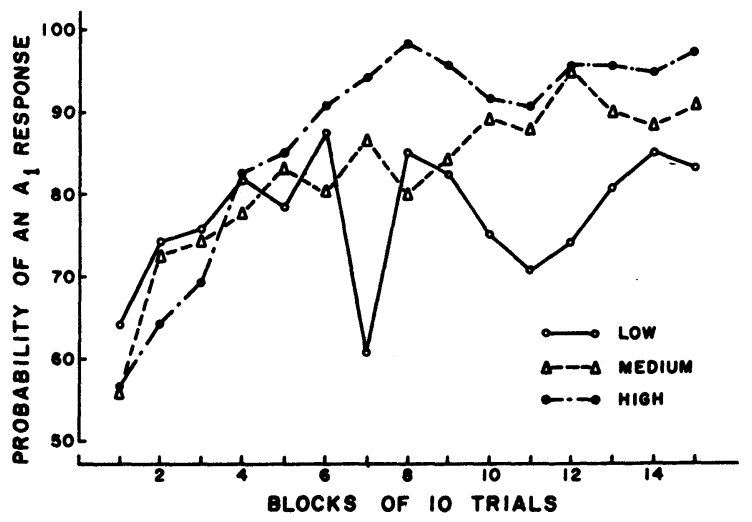

Fig. 1. Leaming curves showing the probability of the more frequently reinforced, $A_{1}$, response as a function of shock intensity. 


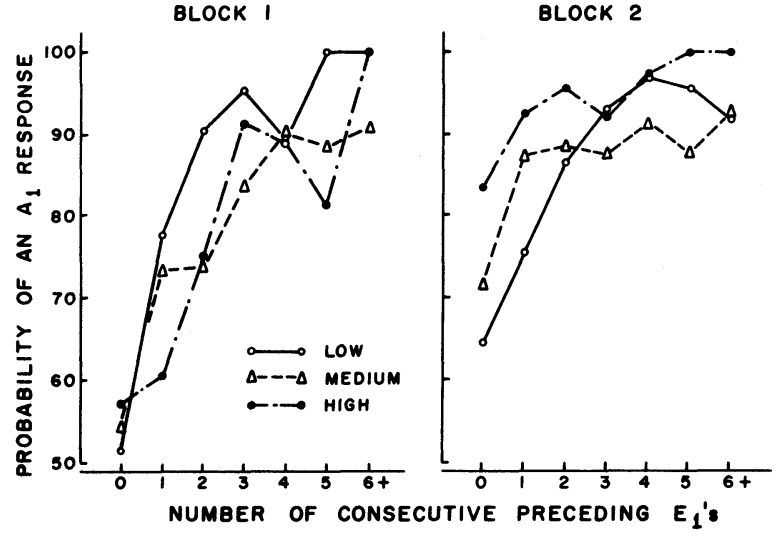

BLOCK 3

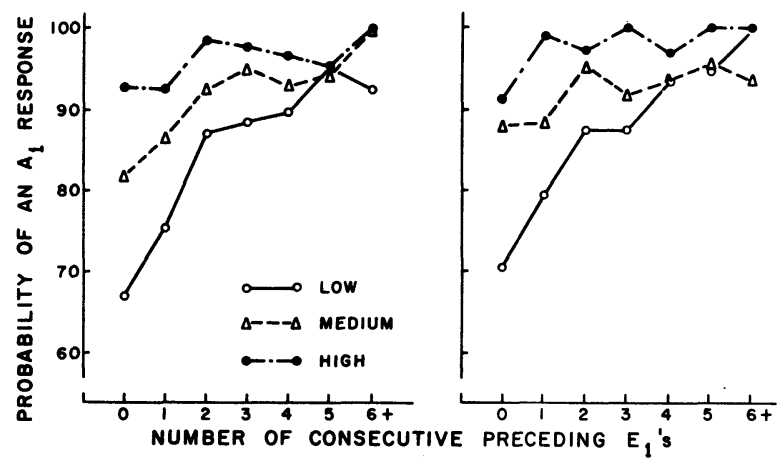

Fig. 2. Recency data: The probability of response $A_{1}$ following various numbers of consecutive $E_{1}$ events is plotted separately for each group. Each sub-graph represents a block of 37 trials (the last two trials are omitted).

$A_{1}$ side, we counted the number of Ss in each group which chose the $\mathrm{A}_{1}$ side on 49 out of the last 50 trials. This number turned out to be 2, 7 and 7 for the low, medium and high groups respectively.

The probability of making the $A_{1}$ response following homogeneous runs of $E_{1}$ events is shown for successive stages of learning in Fig. 2. Two points stand out clearly in the data: a positive recency effect (as defined by Jarvik, 1951) is found at all stages of learning and this effect is at least as strong for the low shock group as it is for the others. Unfortunately, there was insufficient data to determine the analogous functions for $\mathrm{A}_{2}$.
TABLE I

MEAN LATENGIES FOR ALL GROUPS ON THE FOUR POSSIBLE EVENT-RESPONSE CONDITIONS, FOR THE FIRST AND LAST 30 TRIALS

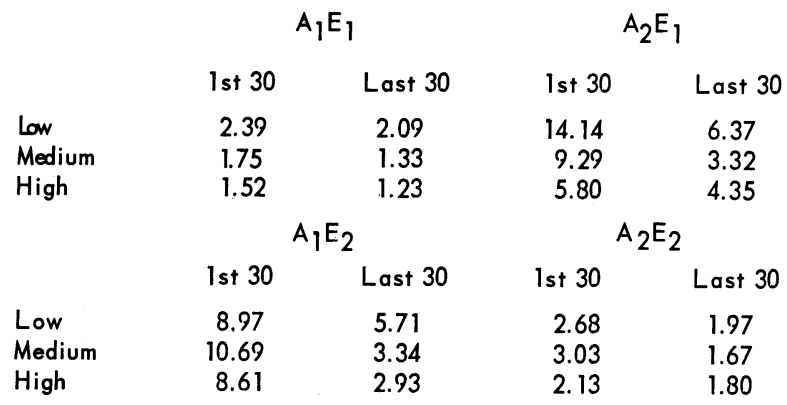

From the latency data presented in Table 1, it appears that response speed is an increasing function of shock level and the $A_{1}$ side is chosen more quickly than $A_{2}$. The latter result is consonant with data presented by Calfee (1963) in an appetitive situation.

Taken as a whole, these data support the contention that amount of reward (as it is reflected in the intensity of shock) combines with reinforcement probability to determine rats' choice behavior in a two choice situation. Theories of choice behavior which hope to account for such behavior must include mechanisms which will predict the degree to which Ss with overshoot $\pi$ as the intensity of the motivational antecedent increases.

\section{Referenees}

Calfee, R. C. Long-term behavior of rats under probabilistic reinforcement schedules. Tech. Rep. No. 59, Institute for Mathematical Studies in the Social Sciences, Stanford University, 1963.

Estes, W. K. The statistical approach to learning theory. In S. Koch (Ed.), Psychology: a study of a science. Vol. II. New York: McGraw-Hill, 1959. Pp. 380-491.

Karsh, E. B., \& Suppes, P. Probability learning of rats in continuous-time experiments. Psychon. Sci., 1964, 1, 361-362.

Myers, J. L., \& Atkinson, R. C. Choice behavior and reward structure. J. math. Psychol., 1964, 1, 170-203.

Uhl, C. H. Two-choice probability learning in the rat as a function of incentive, probability of reinforcement, and training procedure. J. exp. Psychol., 1963, 66, 443-449.

\section{Note}

1. This study was conducted as a part of the senior author's class in learning. Funds for purchase of animals and supplies were supplied by the Department of Psychology, Yale University. 